\title{
Diferencias en la composición florística en bosques de Araucaria- Nothofagus afectados por distintas severidades de fuego
}

\section{Differences in floristic composition of Araucaria-Nothofagus forests affected by mixed levels of fire severity}

\author{
Jonathan Urrutia-Estrada ${ }^{1,2}$, Andrés Fuentes-Ramirez ${ }^{1,3 *}$ \& EnRIQue Hauenstein ${ }^{4}$ \\ ${ }^{1}$ Laboratorio de Biometría, Departamento de Ciencias Forestales, Universidad de La Frontera, casilla 54-D, Temuco, Chile. \\ ²aboratorio de Invasiones Biológicas, Facultad de Ciencias Forestales, Universidad de Concepción, casilla 160-C, \\ Concepción, Chile. \\ ${ }^{3}$ Instituto de Ecología y Biodiversidad (IEB), Santiago, Chile. \\ ${ }^{4}$ Escuela de Ciencias Ambientales, Facultad de Recursos Naturales, Universidad Católica de Temuco, casilla 15-D, Temuco, \\ Chile. \\ *andres.fuentes@ufrontera.cl
}

\begin{abstract}
RESUMEN
El fuego tiene impactos importantes en la estructura y funcionamiento de ecosistemas naturales. En marzo de 2015 un incendio de gran magnitud afectó bosques de alto valor ecológico de Araucaria araucana-Nothofagus pumilio (araucarialenga) en la Reserva Nacional China Muerta (Chile, $38^{\circ} \mathrm{S}$ ), donde las adaptaciones al fuego son poco frecuentes en la flora nativa. El objetivo del presente trabajo fue evaluar los cambios en la composición florística del bosque en un gradiente de severidad de fuego (i.e., alta, media y baja severidad) un año después del disturbio. Se hipotetiza que las zonas más afectadas por el fuego presentarán una composición de especies distinta a la de zonas menos afectadas, con una menor riqueza y abundancia de especies nativas, y que las especies exóticas se verán favorecidas por el fuego poco severo. Se establecieron 20 parcelas de $100 \mathrm{~m}^{2}$ cada una en todo el gradiente de severidad de fuego, incluyendo un bosque adyacente no quemado que sirvió como referencia. En cada parcela se registró la riqueza y abundancia de toda la flora vascular (nativas y exóticas). Se analizó y comparó la riqueza y abundancia de especies, la composición y similitud florística entre los distintos niveles de severidad. Se encontró que la riqueza y abundancia de especies son significativamente más bajas en áreas de alta y media severidad de fuego. La riqueza y abundancia de especies exóticas fue mayor en áreas de baja severidad de fuego, las cuales estuvieron sujetas a pastoreo después del fuego. Existe un rápido cambio composicional de la comunidad de plantas en el bosque de Araucaria-Nothofagus un año después del incendio, sugiriendo un potencial cambio en la estructura del bosque si las especies arbóreas dominantes no se recuperan después del fuego.
\end{abstract}

Palabras clave: Gradiente de severidad, regeneración post-fuego, riqueza de especies, Araucaria araucana, Nothofagus pumilio.

\begin{abstract}
Forest fires can produce significant impacts on the structure and functioning of natural ecosystems. In march 2015, a largescale forest fire affected Araucaria araucana-Nothofagus pumilio (araucaria-lenga) forests of high ecological value in National Reserve China Muerta (Chile, $38^{\circ} \mathrm{S}$ ), where fire adaptations are rare among native plants. The goal of this research was to evaluate the initial changes of plant composition in the forest along a severity gradient (i.e., high, medium and low severity) one year after fire. It is hypothesized that areas more affected by fire will have different species composition from the less-fire-affected areas, with decreased richness and abundance of native species, but exotic species will be favored by the less severe fire. Twenty sampling plots of $100 \mathrm{~m}^{2}$ each were established throughout the fire-severity gradient, including an adjacent unburned forest that served as reference. Species richness and abundance of all vascular plants (native and exotic) were recorded in each plot. We analyzed and compared species richness and abundance of natives and exotics, and the floristic composition and similarity between the different levels of severity. The results show that species richness and abundance were significantly lower in areas of high and medium fire-severity compared to areas less-affected by fire. The richness and abundance of exotic species were greater in areas of low fire-severity, which were subject to cattle grazing after the fire. This research shows a rapid compositional change in the vascular plant community in Araucaria-Nothofagus forests one year after fire, and raises the potential for change in the forest structure if dominant tree species are unable to recover after fire.
\end{abstract}

KEYwORDS: Severity gradient, post-fire regeneration, species richness, Araucaria araucana, Nothofagus pumilio. 


\section{INTRODUCCIÓN}

Las perturbaciones generadas tanto por procesos naturales como derivadas de actividades humanas están entre los factores más importantes que influencian las comunidades ecológicas (Zhang et al. 2014). Bajo escenarios de cambio global, la frecuencia y severidad de perturbaciones (e.g., incendios forestales) pueden ser drásticamente alteradas, y con ello producir un impacto negativo en la biodiversidad (Flannigan et al. 2000). Este fenómeno puede ser más agudo en comunidades poco adaptadas al fuego, pero también puede afectar de manera importante al espectro florístico en ecosistemas que históricamente han co-evolucionado bajo intensos regímenes de incendios (Zhang et al. 2014). En este sentido, aún es necesario avanzar en el desarrollo de un marco teórico general que permita entender la respuesta inicial de la biodiversidad ante los cambios en la frecuencia y severidad del fuego (Supp \& Ernest 2014).

Los bosques templados del sur de Sudamérica no están ajenos al fuego, ya que desde la década de los 80 ha habido un incremento sostenido de incendios forestales, situación que se ha relacionado con el aumento del área plantada de especies forestales exóticas de alta inflamabilidad (e.g., Eucalyptus spp. y Pinus spp.) y otras transformaciones del paisaje (Peña \& Valenzuela 2008, González et al. 2011). Las predicciones climáticas para el centro-sur de Chile son poco alentadoras en cuanto a que se pronostican aumentos de temperatura, una disminución de las precipitaciones y períodos prolongados de sequía (CONAMA 2006). Lo anterior se puede traducir en un probable incremento en la frecuencia y severidad de incendios forestales (González et al. 2011). Esto puede tener graves consecuencias sobre la biodiversidad, ya que los impactos no sólo se traducen en la pérdida inmediata de flora y fauna, sino que también se producen otros fenómenos importantes post-fuego, entre los que destaca el ramoneo de rebrotes de la vegetación (Echeverría et al. 2007), el daño causado por el pisoteo de ganado (Zamorano-Elgueta et al. 2012), el incremento en la llegada de propágulos de especies exóticas invasoras (Pauchard \& Alaback 2004) y el potencial cambio de la composición vegetal (Fuentes-Ramírez et al. 2011).

Si bien la vegetación de los bosques templados del sur de Chile no se caracteriza por presentar adaptaciones al fuego (Montenegro et al. 2004, CONAF 2017), en los ecosistemas andinos dominados por Araucaria araucana (Molina) K. Koch y Nothofagus pumilio (Poepp. \& Endl.) Krasser (de aquí en adelante denominados bosques de AraucariaNothofagus), los incendios forestales han jugado un rol fundamental en la dinámica de estos bosques (González et al. 2005). Sin embargo, los cambios en el régimen del fuego, principalmente derivados de actividades antrópicas, han resultado en incendios más frecuentes y severos en las últimas décadas (González \& Veblen 2006). Históricamente, el régimen de fuego en bosques de Araucaria-Nothofagus ha sido caracterizado por una combinación de incendios superficiales frecuentes (i.e., en promedio cada siete años) de baja severidad con baja mortalidad arbórea e incendios catastróficos poco frecuentes (i.e., en promedio cada 60 años) de alta mortalidad (González et al. 2005). La respuesta post-fuego del bosque se traduce generalmente en la rápida colonización de especies de Nothofagus, tales como $N$. pumilio, N. dombeyi (Mirb.) Oerst. y N. alpina (Poepp. \& Endl.) Oerst., mientras que para A. araucana se presenta un establecimiento más lento y disperso (González \& Veblen 2006). Aunque estas respuestas adaptativas sugieren cierta capacidad de recuperación de los bosques de AraucariaNothofagus después del fuego, aún se desconoce la respuesta de otras especies del sotobosque, principalmente de los arbustos leñosos bajos y las hierbas anuales y perennes. En este contexto, es crucial conocer la respuesta inicial de toda la comunidad vegetal frente a incendios de distintas severidades a fin de poder diseñar e implementar planes de conservación y/o restauración del ecosistema post-fuego.

En ecosistemas donde las especies nativas están poco adaptadas al fuego, las plantas exóticas pueden verse favorecidas por los incendios (D'Antonio 2000, Zouhar et al. 2008). Cuando la frecuencia de incendios es mayor que los regímenes históricos, la vegetación nativa tiende a reducirse, generándose condiciones más favorables para la colonización y establecimiento de especies exóticas (Zouhar et al. 2008), las que posteriormente pueden convertirse en invasoras (Fuentes et al. 2014). Más aun, el fuego puede interactuar positivamente con las hierbas invasoras, promoviendo un mayor establecimiento post-fuego y una mayor acumulación de combustible fino que puede producir incendios más frecuentes y menos severos en el tiempo, lo que a su vez favorece su establecimiento (D'Antonio \& Vitousek 1992). Así, el fuego puede actuar selectivamente excluyendo aquellas especies de plantas que son más sensibles (Hoffmann \& Moreira 2002, Gomes et al. 2014) y promoviendo la homogeneización del ecosistema, lo cual representa una simplificación de la composición de plantas, y consecuentemente reduce la diversidad de especies en el tiempo (Libano \& Felfili 2006).

Los bosques de A. araucana en el sur de Chile representan un patrimonio natural con un alto valor ecológico y social (Aagesen 1998). Esta especie está catalogada como Monumento Natural (Decreto $\mathrm{N}^{\circ} 43$ del Ministerio de Agricultura 1990), lo que prohíbe su corta y explotación forestal. A pesar de su alto valor ecológico y protección legal, actualmente los bosques de $A$. araucana presentan un alto riesgo de degradación, ya sea por tala (González \& Veblen 2006), pastoreo de ganado (FuentesRamírez et al. 2011), sobrexplotación de sus semillas comestibles (piñones) y los efectos inducidos por incendios forestales sobre su composición comunitaria (González et al. 2013, González \& Lara 2015). Además, estos bosques están experimentando procesos recientes de invasión de 
especies exóticas, principalmente por las coníferas Pinus contorta Douglas ex Loudon y P. sylvestris L. (Urrutia et al. 2013, Cóbar-Carranza 2014), y las herbáceas Hypochaeris radicata L., Leontodon taraxacoides Hoppe \& Hornsch., Rumex acetosella L., Taraxacum officinale L. y Trifolium repens L. (Franzese et al. 2017). Es sabido que estas últimas especies herbáceas están fuertemente asociadas a la presencia de ganado (Fuentes-Ramírez et al. 2011) y a perturbaciones humanas como la habilitación de caminos y la degradación del bosque (González et al. 2010, Fuentes et al. 2014). Potencialmente, estas especies exóticas podrían aumentar la frecuencia de incendios poco severos, y con ello, producir cambios irreversibles en la composición y estructura de la vegetación (Keeley 2006, Zouhar et al. 2008).

El objetivo de este estudio es determinar si existen cambios de corto plazo en la riqueza de especies, abundancia y composición florística a través de un gradiente de severidad de fuego (i.e., desde un bosque no afectado por fuego hasta áreas severamente quemadas) en un bosque de Araucaria-Nothofagus y conocer cuáles son las especies de plantas más importantes en la regeneración inicial del bosque post-fuego. Las hipótesis planteadas en este trabajo son: (i) las zonas más afectadas por el fuego (alta y media severidad) presentarán una composición de especies de plantas vasculares distinta a la de zonas menos afectadas por el fuego, con una menor riqueza y abundancia de especies nativas, y (ii) las especies exóticas se verán favorecidas por el fuego poco severo.

Actualmente, existe muy poca información respecto de la relación que existe entre los incendios forestales de diferente severidad y el establecimiento post-fuego de especies exóticas en bosques de Araucaria-Nothofagus en el centro-sur de Chile. Así, este estudio representa un avance significativo en el conocimiento de la respuesta inicial de la vegetación exótica (y potencialmente invasora) con miras a poder desarrollar planes de control y/o manejo de especies invasoras en bosques afectados por incendios forestales.

\section{METODOLOGÍA}

ÁreA DE ESTUDio

El presente trabajo se llevó a cabo en la Reserva Nacional China Muerta, ubicada en la comuna de Melipeuco, Región de La Araucanía ( $38^{\circ} \mathrm{S}, 71^{\circ} \mathrm{W}$; Fig. 1). La Reserva comprende 11.170 ha (CONAF 2014). Presenta una topografía irregular y escarpada, con una altitud que va desde los 800 a los 1.850 m s.n.m. El clima es templado cálido, con menos de 4 meses de sequía (en la temporada estival de diciembre a marzo) y con hielo y nieve debido al efecto de la altitud en los meses de invierno y primavera (junio a octubre; Köppen 1948). La temperatura media en verano es de $19^{\circ} \mathrm{C}$, mientras que en invierno es de $5^{\circ} \mathrm{C}$. La precipitación media anual es de
$2.500 \mathrm{~mm}$. Los suelos del área de estudio corresponden a las series Coyán E y Coyán $\mathrm{F}$, que fueron desarrollados a partir de cenizas volcánicas, con profundidad moderada, de color pardo oscuro (trumaos), con textura gruesa y buena permeabilidad en todo el perfil (CIREN 2010).

El área de estudio pertenece al bosque deciduo andinopatagónico, comprendido en la subregión Cordillera de La Araucanía (Gajardo 1995). La vegetación nativa está representada por los árboles Araucaria araucana, Nothofagus pumilio y $N$. dombeyi. En el estrato arbustivo destacan Chusquea culeou E. Desv., Maytenus disticha (Hook. f.) Urb. y Gaultheria poepigii DC., mientras que en el estrato herbáceo son comunes Osmorhiza chilensis Hook. \& Arn., Viola magellanica G. Forst. y Adenocaulon chilense Less. (Luebert \& Pliscoff 2006). Las especies exóticas más comunes de encontrar son las hierbas Hypochaeris radicata, Cirsium vulgare (Savi) Ten. y Rumex acetosella (González et al. 2010). La Reserva Nacional China Muerta, junto con otras áreas andinas, conforma la Reserva de la Biosfera Araucarias.

\section{DESCRIPCIÓN DEL INCENDIO}

El incendio forestal que afectó la Reserva Nacional China Muerta se inició el 14 de marzo de 2015, siendo controlado 23 días después, el 6 de abril. La superficie total afectada por el fuego, obtenida a través de sobrevuelos desarrollados durante las operaciones de control y con reconocimientos en terreno, alcanzó 3.765 ha, las que excluyen terrenos de altas cumbres descubiertas de vegetación, roqueríos y arenales (CONAF 2015). Después de controlado el incendio, la Corporación Nacional Forestal (CONAF), en conjunto con el Laboratorio de Teledetección Satelital de la Universidad de La Frontera, llevaron a cabo un análisis de imágenes satelitales para determinar el grado de daño del fuego en la vegetación. Usando el índice NBR (normalized burn ratio; Key \& Benson 1999), se determinaron tres niveles de severidad de fuego: alta, media y baja (Fig. 2). Las áreas de alta severidad presentaron un índice NBR $>0,66$, las de media severidad entre 0,6 - 0,35 y las de baja severidad entre 0,35 - 0,1 (Mora \& Crisóstomo 2016). Posteriormente, en terreno se corroboró la clasificación desarrollada por CONAF y cada nivel de severidad se definió de la siguiente manera: El nivel de alta severidad (AS) se caracterizó por presentar un daño completo en el estrato arbóreo, arbustivo y herbáceo, con árboles completamente quemados (desde las raíces hasta las copas) y la vegetación del sotobosque totalmente eliminada a causa del fuego. Además, este nivel presentó una densa capa de cenizas en el suelo (de hasta $30 \mathrm{~cm}$ de profundidad) y grandes agujeros en el suelo causados por la eliminación de tocones de árboles que fueron completamente carbonizados por incendios subterráneos. La severidad media (MS) estuvo caracterizada por zonas donde el fuego quemó parcialmente las copas de los árboles, con el sotobosque también parcialmente dañado por el fuego. Las cenizas sólo 
se depositaron ligeramente en algunas zonas del suelo. Este nivel no presentó evidencias de incendios subterráneos ni eliminación de tocones de árboles. El nivel de baja severidad (BS) correspondió a áreas donde el daño del fuego en las copas de los árboles fue mínimo (sólo porciones menores de algunas ramas fueron quemadas), con abundante follaje verde en las copas y abundante vegetación en el sotobosque. No se encontraron cenizas ni evidencias de incendios subterráneos en este nivel de severidad. Adicionalmente, y como área de referencia, se usó un bosque adyacente donde el fuego no causó ningún tipo de daño a la vegetación
(NBR $<0,1)$, y por lo tanto representa las condiciones de un bosque no quemado (BNQ). En nuestra área de estudio, la condición de BNQ se encuentra distante a $800 \mathrm{~m}$ de las zonas afectadas por el incendio, aproximadamente. Para asegurar la comparabilidad de los datos colectados, nuestro diseño de muestreo fue establecido de tal forma que las variables de sitio fueran similares a través de todo el gradiente de fuego, por lo cual las parcelas levantadas se asemejaban en altitud media (1.425 m s.n.m.), exposición ( $\mathrm{N}$ y NE), pendiente (10$20 \%$ ) y tipo de vegetación (Tabla 1 ).

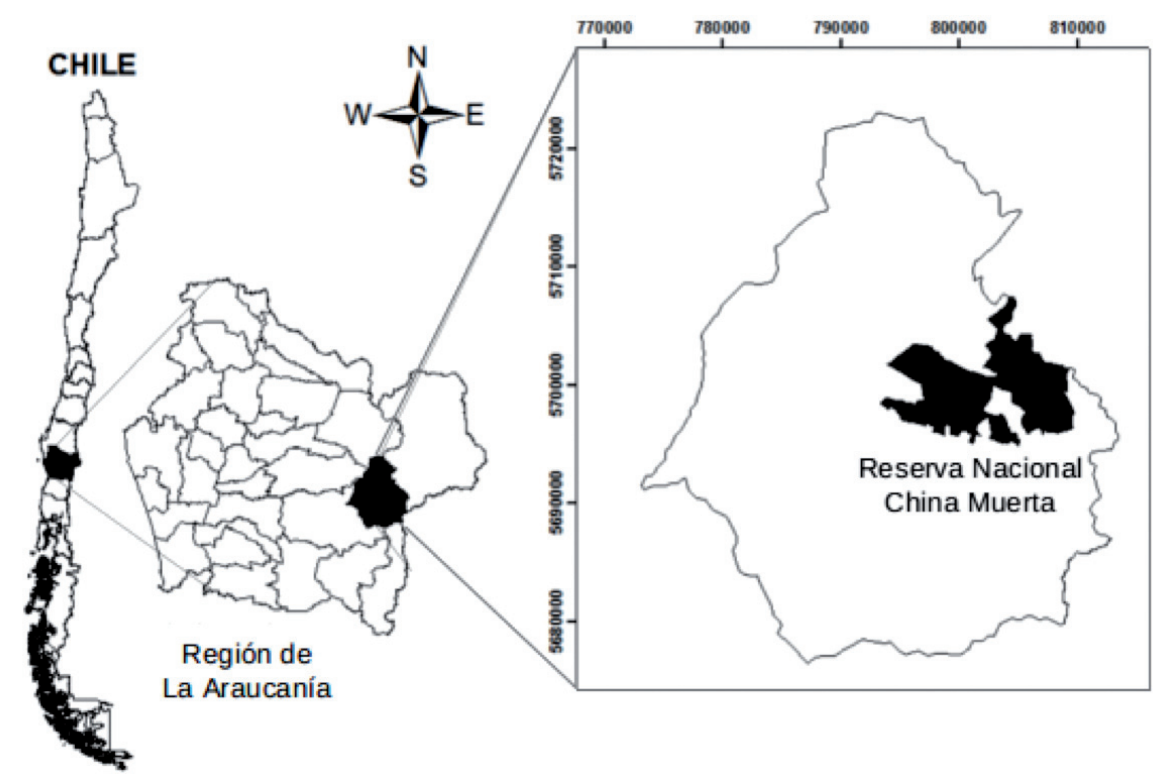

FiguRa 1. Ubicación geográfica del área de estudio en la Reserva Nacional China Muerta, comuna de Melipeuco, Región de La Araucanía $\left(38^{\circ} \mathrm{S}, 71^{\circ} \mathrm{W}\right)$, centro-sur de Chile. / Geographic location of the study area in the China Muerta National Reserve, Melipeuco district, La Araucanía Region $\left(38^{\circ} \mathrm{S}, 71^{\circ} \mathrm{W}\right)$, south-central Chile.
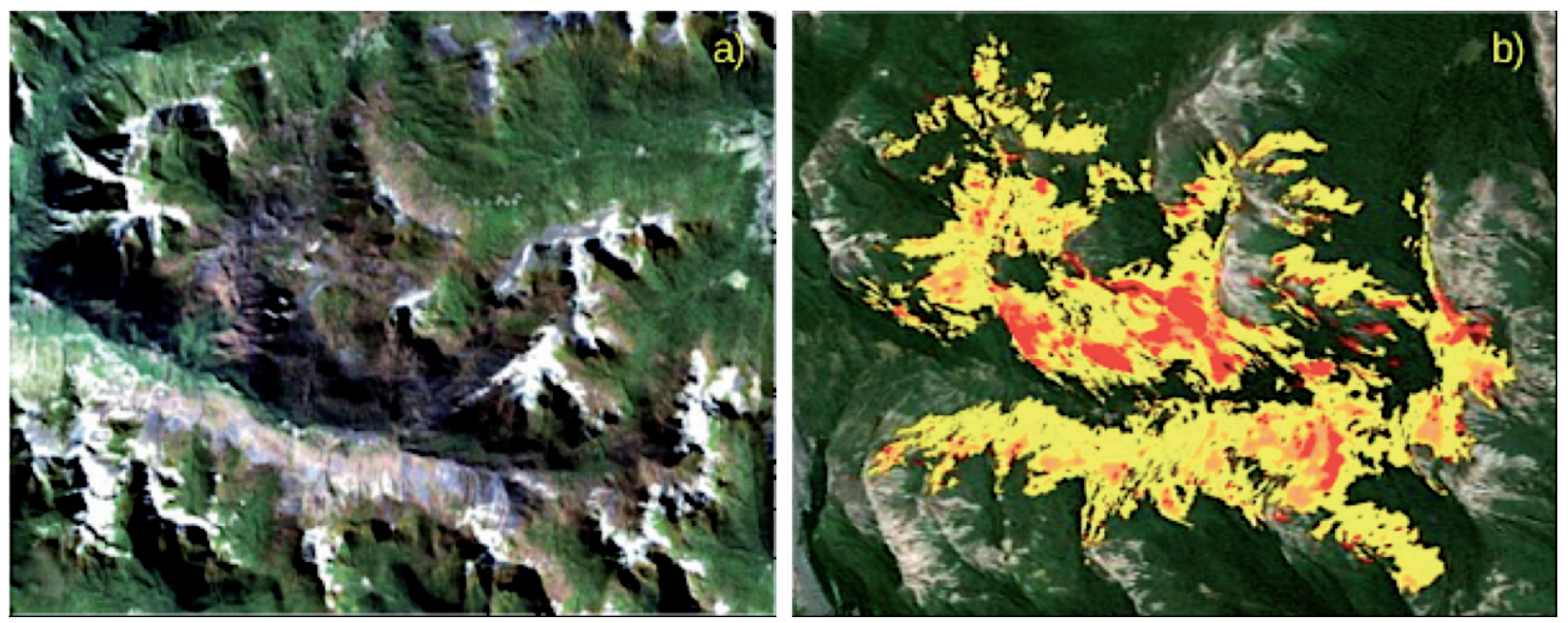

FiguRA 2. a) Imagen aérea mostrando el área quemada en color café oscuro y b) el modelo de severidad de fuego en la Reserva Nacional China Muerta: severidad baja (amarillo), media (naranjo) y alta (rojo). Fuente: Laboratorio de Teledetección Satelital, Departamento de Ciencias Físicas, Universidad de La Frontera. / a) Aerial image showing the burned area in dark brown color and b) the fire-severity model in China Muerta National Reserve: low (yellow), medium (orange) and high (red) severity. Source: Laboratory of Remote Sensing, Department of Physics, Universidad de La Frontera. 
Diferencias florísticas en distintas severidades de fuego: URRUTIA-EstradA, J. ET AL.

TABLA 1. Ubicación geográfica, altitud y exposición de las parcelas de muestreo establecidas en un gradiente de severidad de fuego en la Reserva Nacional China Muerta, Región de La Araucanía. / Geographic location, altitude and exposition for each sample plot established along a burn gradient in China Muerta National Reserve, La Araucanía Region.

\begin{tabular}{|c|c|c|c|c|}
\hline Nivel de severidad & ID parcela & Coordenada geográfica & Altitud (m s.n.m.) & Exposición \\
\hline \multirow{5}{*}{ Alta severidad (AS) } & P01 HS & $38^{\circ} 44.317 \mathrm{~S}-071^{\circ} 31.249 \mathrm{~W}$ & 1.436 & $\mathrm{NE}$ \\
\hline & P02 HS & $38^{\circ} 44.319 \mathrm{~S}-071^{\circ} 31.267 \mathrm{~W}$ & 1.440 & NE \\
\hline & P03 HS & $38^{\circ} 44.326 \mathrm{~S}-071^{\circ} 31.209 \mathrm{~W}$ & 1.426 & NE \\
\hline & P04 HS & $38^{\circ} 44.333 \mathrm{~S}-071^{\circ} 31.130 \mathrm{~W}$ & 1.414 & NE \\
\hline & P05 HS & $38^{\circ} 44.315 \mathrm{~S}-071^{\circ} 31.112 \mathrm{~W}$ & 1.411 & $\mathrm{~N}$ \\
\hline \multirow{5}{*}{ Media severidad (MS) } & P06 MS & $38^{\circ} 44.346 \mathrm{~S}-071^{\circ} 31.102 \mathrm{~W}$ & 1.409 & $\mathrm{NE}$ \\
\hline & P07 MS & $38^{\circ} 44.384 \mathrm{~S}-071^{\circ} 31.040 \mathrm{~W}$ & 1.407 & $\mathrm{~N}$ \\
\hline & P09 MS & $38^{\circ} 44.371 \mathrm{~S}-071^{\circ} 31.020 \mathrm{~W}$ & 1.400 & NE \\
\hline & P11 MS & $38^{\circ} 44.247 \mathrm{~S}-071^{\circ} 31.116 \mathrm{~W}$ & 1.380 & NE \\
\hline & P12 MS & $38^{\circ} 44.215 \mathrm{~S}-071^{\circ} 31.110 \mathrm{~W}$ & 1.375 & NE \\
\hline \multirow{5}{*}{ Baja severidad (BS) } & P08 LS & $38^{\circ} 44.386 \mathrm{~S}-071^{\circ} 31.006 \mathrm{~W}$ & 1.399 & $\mathrm{~N}$ \\
\hline & P10 LS & $38^{\circ} 44.373 \mathrm{~S}-071^{\circ} 30.988 \mathrm{~W}$ & 1.389 & $\mathrm{~N}$ \\
\hline & P13 LS & $38^{\circ} 44.276 \mathrm{~S}-071^{\circ} 31.060 \mathrm{~W}$ & 1.377 & $\mathrm{~N}$ \\
\hline & P14 LS & $38^{\circ} 44.244 \mathrm{~S}-071^{\circ} 31.076 \mathrm{~W}$ & 1.377 & $\mathrm{NE}$ \\
\hline & P15 LS & $38^{\circ} 44.251 \mathrm{~S}-071^{\circ} 31.095 \mathrm{~W}$ & 1.375 & $\mathrm{NE}$ \\
\hline \multirow{5}{*}{$\begin{array}{l}\text { Bosque no quemado } \\
\text { (BNQ) }\end{array}$} & P16 CL & $38^{\circ} 44.031 \mathrm{~S}-071^{\circ} 30.808 \mathrm{~W}$ & 1.467 & NE \\
\hline & P17 CL & $38^{\circ} 44.054 \mathrm{~S}-071^{\circ} 30.835 \mathrm{~W}$ & 1.472 & NE \\
\hline & P18 CL & $38^{\circ} 44.003 \mathrm{~S}-071^{\circ} 30.804 \mathrm{~W}$ & 1.466 & $\mathrm{NE}$ \\
\hline & P19 CL & $38^{\circ} 43.981 \mathrm{~S}-071^{\circ} 30.800 \mathrm{~W}$ & 1.468 & $\mathrm{~N}$ \\
\hline & P20 CL & $38^{\circ} 43.989 \mathrm{~S}-071^{\circ} 30.833 \mathrm{~W}$ & 1.474 & $\mathrm{~N}$ \\
\hline
\end{tabular}

\section{RECOLECCIÓN DE DATOS}

La recolección de datos se realizó un año después de ocurrido el incendio, y comprendió la estación de crecimiento que va desde diciembre de 2015 a abril de 2016, lo que coincide con la época de floración de la mayoría de las especies registradas. Se realizó un levantamiento florístico de toda la vegetación presente en los tres niveles de severidad descritos anteriormente (i.e., AS, MS, BS, e incluyendo el $\mathrm{BNQ}$ ), y que consideró todas las plantas establecidas a partir de semillas o rebrote. Dentro de cada nivel de severidad de fuego se establecieron aleatoriamente cinco parcelas de muestreo permanente de $100 \mathrm{~m}^{2}$ cada una $(10 \times 10 \mathrm{~m}$; $N=20$ ). En cada parcela se registraron todas las especies de plantas vasculares (tanto del estrato arbóreo, arbustivo y herbáceo) y para cada una de ellas se determinó su abundancia total en términos de número de individuos. Para ello se contabilizaron todos los individuos que presentaron una altura $\geq 5 \mathrm{~cm}$ y cuando brotes clonales de una misma especie se separaban a $<5 \mathrm{~cm}$ entre sí, se registraron como un solo individuo. A cada individuo registrado se le asignó un código de identificación y se marcó con una etiqueta metálica permanente para su monitoreo en el tiempo. 
Las especies no identificadas en terreno fueron colectadas en bolsas plásticas, rotuladas y prensadas para su posterior identificación con literatura especializada (Correa 1971, Riedemann \& Aldunate 2004, Teillier et al. 2014). Los especímenes colectados fueron depositados en el herbario de la Universidad Católica de Temuco. Con los datos de vegetación colectados en terreno se elaboró un listado florístico en el cual se detalla el nombre científico de la especie, su autor, familia, origen geográfico, forma de vida y su presencia en cada nivel de severidad de fuego (Anexo 1). La nomenclatura y clasificación taxonómica fue tomada de Zuloaga et al. (2008).

ANÁLISIS DE DATOS

Para comparar la riqueza de especies (exóticas y nativas) en los distintos niveles de severidad de fuego (i.e., BNQ, BS, MS y AS), se realizó una prueba de randomización (con 999 permutaciones) utilizando el paquete estadístico "rich" (Rossi 2011). Este análisis calcula los cuantiles $\mathrm{p}=0,975$ y $\mathrm{p}=0,025$, lo que corresponde a un intervalo global del $95 \%$. Si la diferencia observada en la riqueza de especies es muy superior al valor del cuantil superior, indica que la diferencia observada es mucho mayor de lo esperado bajo la hipótesis nula de "ninguna diferencia entre sitios" (Rossi 2011). Además, se estudió el efecto de la severidad de fuego en la abundancia de plantas mediante un análisis de varianza (ANOVA) de una vía con un test de Tukey a posteriori, lo cual permitió determinar diferencias significativas en la abundancia media entre cada nivel de severidad (con $\alpha=0,05$ ). Para este análisis se revisaron y cumplieron los supuestos de normalidad y homocedasticidad. Tanto el análisis de riqueza de especies como el de abundancia se realizaron en el software estadístico R ( $\mathrm{R}$ Development Core Team 2016).

Posteriormente, y con el fin de comparar la composición florística entre los distintos niveles de severidad de fuego, se realizó un análisis de similitud de una vía (ANOSIM con $\alpha=$ 0,05), para el cual se utilizó el nivel de severidad de fuego como factor predictor. Este análisis realiza comparaciones pareadas y determina diferencias estadísticas para la composición florística entre cada nivel de severidad (i.e., BNQ, BS, MS y AS). Este análisis está basado en una matriz de similitud de Bray-Curtis, el cual se calcula a partir de los datos de riqueza y abundancia de las especies registradas en cada parcela de muestreo.

Finalmente, realizamos un análisis de porcentaje de similitud (SIMPER), el cual permitió establecer la proporción de semejanza florística (y también de disimilitud) entre todos los grupos que presentaron diferencias significativas entre sí, distinguiendo además las especies responsables (según su porcentaje de contribución) de la diferenciación de los grupos según su nivel de severidad. Los análisis de ANOSIM y SIMPER se realizaron con el software Primer-E, versión 6.0 (Clarke \& Gorley 2006).

\section{RESULTADOS}

Se identificó un total de 48 especies de plantas vasculares en todo el gradiente de severidad de fuego, las cuales se reparten en 41 géneros y 29 familias (ver listado florístico completo en Anexo 1). Del total de especies registradas, la mayor riqueza se concentra en BS con 34 especies (28 nativas y 6 exóticas), le siguen el BNQ con 31 especies (30 nativas y 1 exótica), MS con 15 especies (14 nativas y 1 exótica) y finalmente AS con 14 especies (13 nativas y 1 exótica; Fig. 3). Se encontraron diferencias estadísticamente significativas en la riqueza total de especies entre BNQ - MS ( $\Delta=16$ especies; $p=0,001)$, BNQ - AS ( $\Delta=17$ especies; $\mathrm{p}=0,001)$, BS - MS $(\Delta=19$ especies; $\mathrm{p}=0,001)$ y BS $-\mathrm{AS}(\Delta=20$ especies; $\mathrm{p}=0,001)$. La riqueza total de especies no difirió entre BNQ - BS ni entre MS AS (Tabla 2; Fig. 3). Para la riqueza de especies exóticas se encontraron diferencias estadísticamente significativas entre BNQ y BS $(\Delta=5$ especies; $\mathrm{p}=0,01)$, BS y MS $(\Delta=5$ especies; $p=0,02)$, y entre BS y AS $(\Delta=5$ especies; $p=0,01)$. Es interesante destacar que la mayor riqueza y abundancia de especies exóticas se encontró en las zonas de BS (6 especies con un total de 11 individuos) y no en las áreas afectadas por mayor severidad de fuego (AS y MS) (Tabla 2). Las especies exóticas presentes en BS fueron Cirsium arvense (L.) Scop., Holcus lanatus L., Hypochaeris radicata, Lactuca serriola L., Rumex acetosella y Veronica serpyllifolia L.; en BNQ Cerastium arvense L.; en MS Agrostis capillaris L. y en AS R. acetosella (Anexo 1).

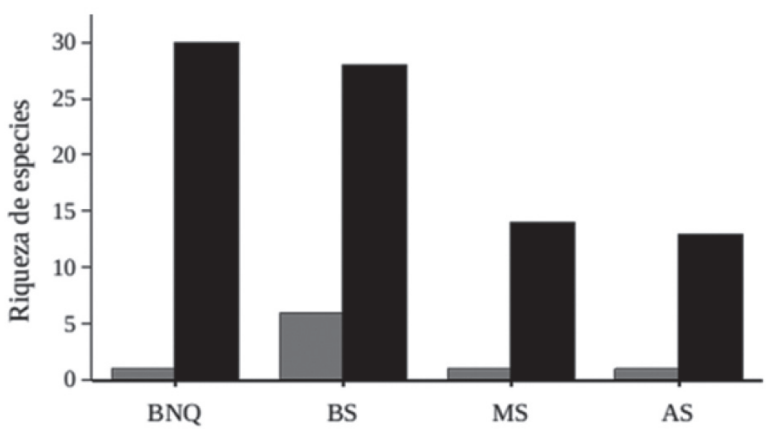

FIgURA 3. Riqueza de especies nativas (barras negras) y exóticas (barras grises) registrada en cada nivel de severidad de fuego. BNQ: bosque no quemado, BS: baja severidad, MS: media severidad, AS: alta severidad. / Species richness for native (black bars) and exotic (gray bars) species within each fire-severity level. BNQ: unburned forest, BS: low severity, MS: medium severity, AS: high severity.

La abundancia media de plantas vasculares a escala de parcela (i.e., en $100 \mathrm{~m}^{2}$ ) resultó ser más de 10 veces mayor en BNQ (476 individuos) que en AS (43 individuos), cuatro veces mayor que en MS (119 individuos) y un poco menos del doble que en BS (283 individuos; Fig. 4). No hubo diferencias significativas en la abundancia media de 
Diferencias florísticas en distintas severidades de fuego: UrRUTIA-Estrada, J. ET AL.

individuos entre AS y MS (Fig. 4). La abundancia total acumulada en todas las parcelas de muestreo reportó un total de 2.382 en BNQ, 1.414 en BS, 528 en MS y 218 individuos en AS.

TABLA 2. Riqueza y abundancia total acumulada (i.e., en todas las parcelas de muestreo) de especies nativas y exóticas para cada nivel de severidad. BNQ: bosque no quemado, BS: baja severidad, MS: media severidad, AS: alta severidad. Note que las distintas letras indican diferencias estadísticamente significativas para la riqueza de especies usando un test de randomización con 999 permutaciones y $\alpha=0,05$. / Species richness and total accumulated abundance (i.e., across all sampling plots) of native and exotic species for each fire-severity level. BNQ: unburned forest, BS: low severity, MS: medium severity, AS: high severity. Note that the different letters indicate statistically significant differences for species richness using a randomization test with 999 permutations and $\alpha=0.05$.

\begin{tabular}{lcccc}
\hline & \multicolumn{2}{c}{ Exóticas } & \multicolumn{2}{c}{ Nativas } \\
\hline & Riqueza & Abund. Total & Riqueza & Abund. Total \\
\hline BNQ & $1 \mathrm{a}$ & 1 & $30 \mathrm{a}$ & 2381 \\
BS & $6 \mathrm{~b}$ & 11 & $28 \mathrm{a}$ & 1403 \\
MS & $1 \mathrm{a}$ & 2 & $14 \mathrm{~b}$ & 526 \\
AS & $1 \mathrm{a}$ & 3 & $13 \mathrm{~b}$ & 215 \\
\hline
\end{tabular}

Como especies comunes en todo el gradiente de fuego, es decir, que se presentaron en los cuatro niveles de severidad, aparecen Alstroemeria aurea Graham, Araucaria araucana, Berberis microphylla G. Forst., Chusquea culeou, Gaultheria poeppigii y Vicia nigricans Hook. \& Arn. En tanto, como especies únicas (i.e., que están presentes sólo en un nivel de severidad) se registraron Cerastium arvense, Codonorchis lessonii (Brongn.) Lindl., Galium hypocarpium (L.) Endl., Lagenophora hariotii Franch., Perezia pedicularidifolia Less., Quinchamalium chilense Molina y Sisyrinchium arenarium Poepp. sólo en BNQ; Acaena pinnatifida

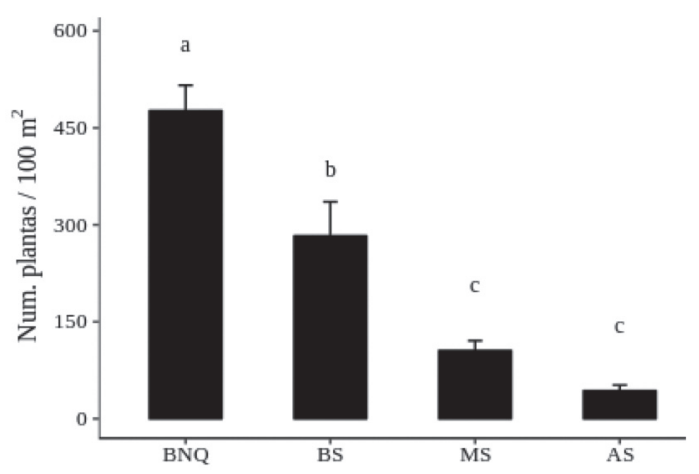

FIGURA 4. Abundancia media de plantas vasculares por parcela $\left(100 \mathrm{~m}^{2}\right)$ según severidad de fuego. BNQ: bosque no quemado, BS: baja severidad, MS: media severidad y AS: alta severidad. Note que diferentes letras indican diferencias estadísticamente significativas para $\alpha=0,05$ en la abundancia media de plantas vasculares calculada con la prueba de Tukey. / Mean abundance of vascular plants per plot $\left(100 \mathrm{~m}^{2}\right)$ according to fire severity levels. BNQ: unburned forest, BS: low severity, MS: moderate severity and AS: high severity. Note that different letters indicate statistically significant differences for $\alpha=0.05$ in mean abundance of vascular plants calculated with the Tukey test.
Ruiz \& Pav., Anemone antucensis Poepp., Calceolaria valdiviana Phil., Cirsium arvense, Euphorbia peplus L., Lactuca serriola, Perezia nutans Less., Phacelia secunda J.F. Gmel., y Ribes cucullatum Hook. \& Arn. sólo en BS; y Adesmia emarginata Clos, Baccharis magellanica (Lam.) Pers. y Rodophiala andicola (Poepp.) Traub sólo en AS. Es importante destacar que no se registraron especies únicas para el nivel MS.

Las especies más abundantes que rebrotaron en todos los niveles de severidad de fuego (i.e., BNQ, BS, MS y AS) fueron M. disticha, G. poeppigii, C. culeou, N. pumilio y A. aurea. Estas cinco especies por sí solas representaron más del $65 \%$ de toda la abundancia de la vegetación postfuego. La abundancia de $A$. araucana fue más de 13 veces mayor en BNQ (106 individuos) que en las áreas afectadas por el fuego (8 individuos en BS, MS y AS). Por otro lado, $N$. pumilio, la otra especie arbórea dominante, estuvo completamente ausente de las áreas de AS.

El ANOSIM reveló la existencia de diferencias estadísticamente significativas para la composición florística entre los distintos niveles de severidad de fuego estudiados ( $\mathrm{R}$ global $=0,672 ; \mathrm{p}=0,0003)$. La composición florística fue significativamente distinta entre BNQ y $B S(p=0,008)$, entre BNQ y MS ( $p=0,008)$, BNQ y AS $(p=0,008)$, BS y MS ( $p=0,008)$, y BS y AS ( $p=0,008$; Tabla 3$)$. El análisis SIMPER indicó que la mayor similitud intragrupos ocurre en la condición de BNQ con un $60,9 \%$, en tanto que la más baja se da en el nivel AS con sólo un 37,5\% de similitud florística. Del mismo modo, al realizar las comparaciones inter-grupos entre los niveles de severidad que las comparaciones pareadas señalaron como significativamente diferentes, encontramos que la mayor disimilitud florística ocurre entre BNQ y AS con un $88,4 \%$. Le siguen BS y AS con $81,5 \%$, BNQ y MS con $81,1 \%$, BS y MS con $69,2 \%$ y finalmente BNQ y BS con un $54,7 \%$ de disimilitud florística (Tabla 4). 
TABLA 3. Análisis de similitud florística ANOSIM con comparaciones pareadas entre todos los niveles de severidad de fuego estudiados. BNQ: bosque no quemado, BS: baja severidad, MS: media severidad, AS: alta severidad. / ANOSIM similarity analysis with pair-wise comparisons between all fire-severity levels included in the study. BNQ: unburned forest, BS: low severity, MS: medium severity, AS: high severity.

\begin{tabular}{lcc}
\hline Comparación & Estadístico R & VAlor P \\
\hline BNQ - AS & 0,996 & 0,008 \\
BNQ - BS & 0,696 & 0,008 \\
BNQ - MS & 0,852 & 0,008 \\
AS - BS & 0,880 & 0,008 \\
AS - MS & 0,128 & 0,151 \\
BS - MS & 0,648 & 0,008 \\
\hline
\end{tabular}

TABLA 4. Análisis de porcentaje de similitud (SIMPER) entre todos los grupos que presentaron diferencias significativas entre sí, según comparaciones pareadas. BNQ: bosque no quemado, BS: baja severidad, MS: media severidad, AS: alta severidad. Ab. Prom.: abundancia promedio, Dis. Prom.: disimilitud promedio, DE: desviación estándar, Con.: contribución, Acu.: acumulado. / Analysis of similarity percentage (SIMPER) between all groups that showed statistically significant differences for their paired-comparisons. BNQ: unburned forest, BS: low severity, MS: medium severity, AS: high severity. Ab. Prom.: average abundance, Dis. Prom.: average dissimilarity, DE: standard deviation, Con.: contribution, Acu.: accumulated.

\begin{tabular}{|c|c|c|c|c|c|c|}
\hline \multirow{2}{*}{$\begin{array}{l}\text { Especies } \\
\text { Prom. Disim: 88,37\% }\end{array}$} & \multicolumn{2}{|c|}{ Ab. Prom. } & \multirow[t]{2}{*}{ Dis. Prom. } & \multirow[t]{2}{*}{ Dis./DE } & \multirow[t]{2}{*}{ Con. (\%) } & \multirow[t]{2}{*}{ Acu. $(\%)$} \\
\hline & BNQ & AS & & & & \\
\hline Maytenus disticha & 12,55 & 0,00 & 16,24 & 3,18 & 18,38 & 18,38 \\
\hline Gaultheria poeppigii & 9,57 & 2,50 & 10,15 & 1,91 & 11,48 & 29,86 \\
\hline Adenocaulon chilense & 5,28 & 0,00 & 6,74 & 2,56 & 7,63 & 37,48 \\
\hline Nothofagus pumilio & 5,00 & 0,00 & 5,87 & 1,67 & 6,64 & 44,12 \\
\hline Ribes magellanicum & 4,62 & 0,00 & 5,82 & 7,06 & 6,58 & 50,71 \\
\hline Prom. Disim: 81,54\% & AS & $\mathrm{BS}$ & & & & \\
\hline Maytenus disticha & 0,00 & 7,98 & 11,78 & 6,65 & 14,45 & 14,45 \\
\hline Nothofagus pumilio & 0,00 & 6,02 & 8,33 & 2,23 & 10,21 & 24,67 \\
\hline Berberis serratodentata & 0,00 & 5,49 & 8,28 & 1,98 & 10,16 & 34,83 \\
\hline Alstroemeria aurea & 1,74 & 5,05 & 5,25 & 1,86 & 6,43 & 41,26 \\
\hline Acaena ovalifolia & 0,00 & 3,27 & 4,78 & 2,14 & 5,86 & 47,12 \\
\hline Ribes magellanicum & 0,00 & 3,22 & 4,38 & 2,94 & 5,37 & 52,49 \\
\hline Prom. Disim: 81,08 & BNQ & MS & & & & \\
\hline Maytenus disticha & 12,55 & 1,07 & 13,53 & 2,73 & 16,68 & 16,68 \\
\hline Gaultheria poeppigii & 9,57 & 1,81 & 9,62 & 1,71 & 11,87 & 28,55 \\
\hline Chusquea culeou & 0,98 & 7,37 & 7,95 & 1,60 & 9,80 & 38,35 \\
\hline Adenocaulon chilense & 5,28 & 0,00 & 6,11 & 2,60 & 7,53 & 45,89 \\
\hline Nothofagus pumilio & 5,00 & 0,82 & 4,94 & 1,52 & 6,10 & 51,98 \\
\hline Prom. Disim: $69,24 \%$ & $\mathrm{BS}$ & MS & & & & \\
\hline Maytenus disticha & 7,98 & 1,07 & 9,16 & 3,17 & 13,22 & 13,22 \\
\hline Chusquea culeou & 1,46 & 7,37 & 8,70 & 1,91 & 12,56 & 25,78 \\
\hline Berberis serratodentata & 5,49 & 0,40 & 6,83 & 1,81 & 9,87 & 35,65 \\
\hline Nothofagus pumilio & 6,02 & 0,82 & 6,78 & 1,84 & 9,79 & 45,44 \\
\hline Gaultheria poeppigii & 3,34 & 1,81 & 4,05 & 1,23 & 5,85 & 51,29 \\
\hline Prom. Disim: $54,74 \%$ & BNQ & $\mathrm{BS}$ & & & & \\
\hline Gaultheria poeppigii & 9,57 & 3,34 & 6,29 & 1,67 & 11,49 & 11,49 \\
\hline Berberis serratodentata & 0,28 & 5,49 & 4,37 & 1,80 & 7,98 & 19,47 \\
\hline Maytenus disticha & 12,55 & 7,98 & 4,03 & 1,41 & 7,36 & 26,83 \\
\hline Alstroemeria aurea & 0,75 & 5,05 & 3,55 & 2,34 & 6,48 & 33,31 \\
\hline Adenocaulon chilense & 5,28 & 1,89 & 3,15 & 1,43 & 5,76 & 39,07 \\
\hline Nothofagus pumilio & 5,00 & 6,02 & 3,13 & 1,29 & 5,71 & 44,79 \\
\hline Myoschilos oblonga & 3,19 & 0,20 & 2,47 & 1,57 & 4,52 & 49,30 \\
\hline Acaena ovalifolia & 0,49 & 3,27 & 2,41 & 1,80 & 4,41 & 53,71 \\
\hline
\end{tabular}




\section{DISCUSIÓN}

Un año después del incendio ocurrido en la Reserva Nacional China Muerta, la riqueza de especies de plantas vasculares que presentan los sitios más afectados por el fuego (AS y MS) es considerablemente menor a la que se presenta en áreas menos afectadas (BS) y bosque no quemado (BNQ). Además de ser menor, la composición florística varía entre sitios en función de la severidad. Estos resultados sustentan nuestra primera hipótesis de trabajo, respecto de que el fuego es un modelador importante de la composición de especies vegetales en bosques de Araucaria-Nothofagus en los Andes del sur de Chile.

La baja riqueza de especies post-incendio en las zonas de alta y media severidad puede deberse en gran medida a la alta intensidad del fuego que quemó completamente el sotobosque y los árboles dominantes (A. araucana y $N$. pumilio). Las especies que más lograron regenerar en dichas zonas son aquellas que no sufrieron daños significativos en su sistema radicular y que poseen además una alta capacidad de rebrote vegetativo, estrategia beneficiosa bajo condiciones de fuego (Pfab \& Witkowski 1999, Bond \& Midgley 2003, González et al. 2010). Entre estas especies destacan C. culeou, G. poeppigii y A. aurea. Por otro lado, la riqueza total de especies en zonas afectadas por baja severidad de fuego (BS) es muy similar a la riqueza encontrada en el bosque adyacente no quemado (33 vs. 31 especies, respectivamente). Nuestros resultados son consistentes con otros estudios que han examinado el efecto de la severidad del fuego sobre la riqueza de especies en ecosistemas forestales. En general, se muestra que la riqueza de especies disminuye a medida que la severidad del fuego aumenta (Morgan et al. 2015, Blair et al. 2016). Además, se ha demostrado que los incendios severos pueden suprimir cada vez más aquellas especies más sensibles al fuego (Hoffmann \& Moreira 2002). Finalmente, esto puede conducir a una disminución sostenida de la riqueza de especies, y en última instancia, a un bosque menos diverso y simplificado con el paso del tiempo (Eskuche 2002).

Respecto de los cambios en la composición de especies en zonas afectadas por distinta severidad de fuego, nuestra investigación reporta que del total de especies registradas en todo el gradiente de severidad de fuego, 17 y 15 de estas especies fueron excluidas en áreas de AS y MS, respectivamente. Esto representa una reducción de más del $50 \%$ de la riqueza en comparación con los bosques no quemados (BNQ). Entre las especies temporalmente excluidas de las áreas de alta severidad de fuego se encuentran N. pumilio, Ribes trilobum Meyen, Adenocaulon chilense, Codonorchis lessonii y Uncinia scabriuscula G.A. Wheeler. Estas especies serían más sensibles a fuegos de alta severidad, al carecer de respuestas adaptativas a los fuegos severos (i.e., capacidad de rebrote o germinación post-fuego), y por ende, con escasas posibilidades de establecerse tras un incendio de alta severidad. En tal sentido, una reducción tan significativa de la riqueza de especies en áreas afectadas por fuego severo puede representar una simplificación de la comunidad vegetal y una reducción de la diversidad biológica en los bosques de Araucaria-Nothofagus.

En relación a las especies exóticas, nuestro estudio mostró un bajo número de éstas en áreas de $\mathrm{AS}$ y MS, comparable incluso con BNQ, sugiriendo que el fuego severo no favorecería el establecimiento de estas especies, al menos en el corto plazo (i.e., un año después del incendio). De hecho, las diferencias de composición detectadas por el ANOSIM entre BQN y BS están determinadas principalmente por la mayor proporción de especies exóticas en esta última condición. En áreas de BS se observó un incremento significativo de la riqueza y abundancia de especies exóticas, lo que sustenta nuestra segunda hipótesis de trabajo en relación a que las especies exóticas se verían favorecidas por fuegos de baja severidad. En las zonas de BS, además de haber sido afectadas por fuego, se observó la presencia de ganado vacuno, el cual puede actuar como vector de dispersión de especies exóticas procedentes de otras áreas (Chaichi et al. 2005, Savadogo et al. 2007, Amiri et al. 2008). De hecho, algunas de las especies exóticas identificadas en BS son indicadoras de compactación de suelos (usualmente asociada al pisoteo de ganado), tales como Lactuca serriola y Cirsium arvense (Matthei 1995, Fuentes et al. 2014). La presencia de herbívoros, como el ganado, puede producir cambios en la composición de especies de la comunidad vegetal, abriendo claros en la vegetación y favoreciendo el establecimiento de hierbas anuales (Sinkins \& Otfinowski 2012, Chuong et al. 2016). Esto produce una reducción en la carga de combustible, aumentando la probabilidad de ocurrencia de incendios de baja severidad, lo que a su vez favorece el establecimiento y la permanencia en el tiempo de las hierbas invasoras (D'Antonio \& Vitousek 1992, Raffaele et al. 2011). En tal sentido, no es posible descartar que las zonas expuestas a baja severidad ya presentaran una alta proporción de hierbas exóticas (asociadas a la ganadería) antes del incendio. Dichas especies, en general, contribuyen con una baja carga de combustible, lo que pudo haber determinado que el fuego haya sido menos severo que en las otras zonas del estudio.

Interesantemente, algunas especies nativas pudieron rebrotar en abundancia en las zonas de fuego severo (AS), como por ejemplo, Chusquea culeou, Gaultheria poeppigii y Alstroemeria aurea. Los representantes del género Chusquea se caracterizan por poseer un sistema de rizomas, el cual favorece su aparición en el proceso sucesional inicial después del fuego (González et al. 2010). De igual manera, el rebrote vegetativo de G. poeppigii se debe a una característica propia de las formas de vida ericoides, las cuales tienen una alta capacidad de rebrote luego de eventos de remoción de biomasa aérea, tal como ocurre en incendios 
forestales (Le Maitre et al. 1992), esto principalmente a través de rebrotes del lignotuber (Ojeda et al. 2016). Por último, para $A$. aurea se ha descrito la presencia de simbiosis con micorrizas del tipo arbuscular, lo cual podría conducir a una rápida colonización por rebrote en los sitios afectados por fuego (Godoy et al. 1994). Además, se debe considerar que por el hecho de corresponder a una planta de hábito geófito, el fuego estimula su crecimiento y floración postdisturbio (Lamont \& Downes 2011). Así, especies con buena capacidad de rebrote (como las señaladas anteriormente) tendrían ventajas para establecerse después de fuegos severos en bosques de Araucaria-Nothofagus (González et al. 2010).

Si bien el área de estudio no ha sido afectada por incendios de gran magnitud en su historia reciente (e.g., últimos 60 años), se predice que para el centro-sur de Chile eventos de este tipo se tornarán cada vez más frecuentes y severos (Ubeda \& Sarricolea 2016). En tal sentido, se debe señalar que incendios sucesivos tienden a promover el establecimiento de especies exóticas incluso en ecosistemas donde las especies nativas están adaptadas a incendios naturales (D'Antonio \& Vitousek 1992, Keeley 2006, Zouhar et al. 2008). En el caso de los bosques del centro-sur de Chile, dado que las especies nativas no suelen responder de forma favorable al fuego (Gómez-González et al. 2017, Molina et al. 2017), éstas podrían verse considerablemente afectadas, y eventualmente llegar a ser desplazadas o experimentar extinciones locales (Mack \& D'Antonio 1998, Brooks et al. 2004). Por ejemplo, nuestros resultados indican que la abundancia de A. araucana y $N$. pumilio, especies arbóreas dominantes y emblemáticas de los bosques del sur de Chile, puede reducirse drásticamente tras incendios severos. Además, N. pumilio podría encontrar dificultades para recuperarse después de incendios forestales de alta severidad, ya que necesita de una fuente de semillas cercana y también un estrato arbóreo que promueva y proteja su establecimiento (González et al. 2006). La reducción en la abundancia de estas especies arbóreas dominantes podría incluso afectar el desarrollo de la estructura del bosque y modificar su vulnerabilidad al fuego en el largo plazo (Paritsis et al. 2015, Kitzberger et al. 2016). Así, la recuperación natural de estos bosques dependerá en gran medida de las especies colonizadoras que lleguen primero a ocupar el sitio (Egler 1954), de la capacidad de regeneración de las plantas y de los procesos de invasión que pudieran darse después del fuego, entre otros factores (Pickett et al. 1987, Brooks et al. 2004).

\section{RESTAURACIÓN EN ÁREAS QUEMADAS}

A la luz de los resultados presentados en este trabajo, de esta investigación se pueden extraer ideas relevantes que pueden ser de utilidad en el diseño e implementación de planes manejo y/o restauración al interior de la Reserva Nacional China Muerta, específicamente para (i) evitar la propagación de especies exóticas invasoras post-fuego y (ii) restaurar las zonas afectadas con mayor severidad de fuego, donde las especies arbóreas dominantes (A. araucana y $N$. pumilio) resultaron significativamente afectadas. Para el primer caso, una recomendación inmediata es tratar de reducir la dispersión de propágulos de especies exóticas en áreas afectadas por fuego, las cuales son frecuentemente dispersadas por el pastoreo. En este sentido, limitar la entrada de ganado hacia áreas de mayor invasibilidad sería una medida efectiva en la disminución del riesgo de invasiones de plantas exóticas post-fuego (Amiri et al. 2008). Además, en aquellas zonas donde se ha observado una alta tasa de reclutamiento y rebrote de las especies arbóreas mencionadas anteriormente, se pueden delimitar áreas de recuperación y excluirlas tanto del uso ganadero, recreacional y otros, como la recolección de piñones. En el caso de $N$. pumilio, se recomiendan técnicas asistidas para recuperar su cobertura, ya que desapareció completamente en áreas de alta severidad de fuego. Existen metodologías novedosas que han dado buenos resultados en términos de porcentaje de prendimiento y crecimiento para la restauración de otras especies nativas arbóreas (Bannister 2015), como la plantación en grupos (i.e., cluster planting; Saha et al. 2017), la cual puede ser aplicada en el área de estudio y complementada con la delimitación de áreas de recuperación descrita anteriormente.

\section{CONCLUSIONES}

A través de esta investigación encontramos un rápido cambio composicional de la comunidad de plantas vasculares en bosques de Araucaria-Nothofagus afectados por un incendio forestal con distintos grados de severidad de fuego en la Reserva Nacional China Muerta. La composición de especies de plantas vasculares que presentan los sitios más afectados por el fuego (AS y MS) es significativamente menor y distinta a la que se presenta en áreas menos afectadas (BS) y en bosque no quemado (BNQ). En relación a las especies exóticas, nuestro estudio reporta un bajo número de éstas en áreas de AS y MS, no así en BS, donde el fuego parece favorecer la colonización de especies exóticas en el corto plazo. La interacción entre el fuego de baja severidad con otras perturbaciones como el pastoreo podría potenciar el establecimiento de especies exóticas, y con ello el proceso de invasión en el tiempo. Tanto $A$. araucana como $N$. pumilio (las dos especies arbóreas dominantes) se vieron significativamente afectadas por el fuego de alta severidad, lo que podría generar un cambio en la estructura del bosque si estas especies no son capaces de recuperarse después del fuego. Las estrategias de manejo deben enfocarse principalmente en prevenir la propagación de especies exóticas al interior de áreas quemadas y en la recuperación de $N$. pumilio, especie representativa de 
Diferencias florísticas en distintas severidades de fuego: URRUTIA-EstradA, J. ET AL.

los bosques del sur de Chile y altamente vulnerable a los incendios severos.

\section{AGRADECIMIENTOS}

Esta investigación fue financiada por el Proyecto FONDECYT Iniciación $\mathrm{N}^{\circ}$ 11150487, Ministerio de Educación, Chile. AFR agradece el apoyo recibido del Concurso Nacional Inserción en la Academia 2017, CONICYT-PAI 79170054 y de la Vicerrectoría de Investigación y Postgrado (VRIP) de la Universidad de La Frontera. Los autores agradecen a la Corporación Nacional Forestal (CONAF), a los guardaparques de la Reserva Nacional China Muerta y a Marcia Barrientos, Paola Arroyo, Andrea del Fierro y Franco Pérez por su colaboración en el trabajo de terreno.

\section{REFERENCIAS}

AagesEn, D.L. 1998. Indigenous resource rights and conservation of the Monkey-Puzzle tree (Araucaria araucana, Araucariaceae): A case study from southern Chile. Economic Botany 52: 146-160.

Amiri, F., AriaPOUR, A., FADAI, S. 2008. Effects of livestock grazing on vegetation composition and soil moisture properties in grazed and non-grazed range site. Journal of Biological Sciences 8: 1289-1297.

BANNISTER, J.R. 2015. Recuperar bosques no es sólo plantar árboles: Lecciones aprendidas luego de 7 años restaurando bosques de Pilgerodendron uviferum (D. Don) Florin en Chiloé. Anales del Instituto de la Patagonia 43: 35-51.

Blair, D.P., McBurney, L.M., Blanchard, W., Banks, S.C., LindenMaYer, D.B. 2016. Disturbance gradient shows logging affects plant functional groups more than fire. Ecological Applications 26: 2280-2301.

Bond, W., Midgley, J. 2003. The evolutionary ecology of sprouting in woody plants. International Journal of Plant Sciences 164: 103-114.

Brooks, M.L., D’Antonio, C.M., Richardson, D.M., Grace, J.B., Keeley, J.E., DiTomaso, J.M., Hobbs, R.J., Pellant, M. Pyke, D. 2004. Effects of invasive alien plants on fire regimes. BioScience 54: 677-688.

Centro de Información de Recursos Naturales (CIREN). 2010. Determinación de la erosión actual y potencial de los suelos de Chile. CIREN, Santiago, Chile. 145 pp.

Chaichi, M., Saravi, M., Malekian, A. 2005. Effects of livestock trampling on soil physical properties and vegetation cover (Case study: Lar Rangeland, Iran). International Journal of Agriculture \& Biology 7: 904-908.

Chuong, J., Huxley, J., Spotswood, E.N., Nichols, L., Mariotte, P., Suding, K.N. 2016. Cattle as dispersal vectors of invasive and introduced plants in a California annual grassland. Rangeland Ecology \& Management 69: 52-58.

Clarke, K., Gorley, R. 2006. Plymouth routines in multivariate ecological research. Primer-E. Plymouth Marine Laboratory, London, UK. 190 pp.
Cóbar-Carranza, A., García, R.A., Pauchard, A., Peña, E. 2014. Effect of Pinus contorta invasion on forest fuel properties and its potential implications on the fire regime of Araucaria araucana and Nothofagus antarctica forests. Biological Invasions 16: 2273-2291.

Comisión Nacional del Medio Ambiente (CONAMA). 2006. Estudio de la variabilidad climática en Chile para el siglo XXI. Informe final. CONAMA, Santiago, Chile. 71 pp.

Corporación Nacional Forestal (CONAF). 2014. Plan de manejo Reserva Nacional China Muerta. Documento operativo. Sistema Nacional de Áreas Silvestres Protegidas del Estado, Santiago, Chile. 73 pp.

Corporación Nacional Forestal (CONAF). 2015. Plan de restauración incendio Reserva Nacional China Muerta. CONAF, Temuco, Chile. 64 pp.

Corporación Nacional Forestal (CONAF). 2017. Ocurrencia nacional de incendios forestales por región: Periodo 19772016. Gerencia Manejo del Fuego, Santiago, Chile.

Correa, M. 1971. Flora patagónica. Parte VII. Compositae. Instituto Nacional de Tecnología Agropecuaria (INTA), Buenos Aires, Argentina. $451 \mathrm{pp}$.

D'Antonio, C.M. 2000. Fire, plant invasions, and global changes. In: Mooney, H., HobBs, R.J. (eds.), Invasive species in a changing world, pp. 65-95. Island Press, Washington D.C., USA.

D’Antonio, C.M., Vitousek, P.M. 1992. Biological invasions by exotic grasses, the grass/fire cycle, and global change. Annual Review of Ecology and Systematics 23: 63-87.

Echeverria, C., Newton, A.C., Lara, A., Benayas, J.M.R., Coomes, D.A. 2007. Impacts of forest fragmentation on species composition and forest structure in the temperate landscape of southern Chile. Global Ecology and Biogeography 16: 426-439.

EGLER, F. 1954. Vegetation science concepts I. Initial floristic composition, a factor in old-field vegetation development. Vegetatio 4: 412-417.

Eskuche, U. 2002. Pflanzensociolgische Untersuchungen in Nordpatagonien. IV. Die Wälder des Nothofagion pumilionis. Folia Botanica et Geobotanica Correntesiana 16: 1-47.

Flannigan, M.D., Stocks, B.J., Wotton, B.M. 2000. Climate change and forest fires. Science of the Total Environment 262: 221-229.

Franzese, J., Urrutia, J., García, R., Taylor, K., Pauchard, A. 2017. Pine invasion impacts on plant diversity in Patagonia: Invader size and invaded habitat matter. Biological Invasions 19: 1015-1027.

Fuentes, N., Sánchez, P., Pauchard, A., Urrutia, J., Cavieres, L., Marticorena, A. 2014. Plantas invasoras del centro-sur de Chile. Una guía de campo. Laboratorio de Invasiones Biológicas, Universidad de Concepción, Concepción, Chile. 280 pp.

Fuentes-Ramírez, A., Pauchard, A., Hauenstein, E. 2011. Floristic composition of Andean grassland in Alto Bio-Bio National Reserve (Lonquimay-Chile) and its relationship with the grazing regimen. Gayana Botánica 68: 28-39.

Gajardo, R. 1995. La vegetación natural de Chile. Clasificación y distribución geográfica. Editorial Universitaria, Santiago, Chile. 165 pp.

Godoy, R., Romero, R., Carrillo, R. 1994. Estatus micotrófico de 
la flora vascular en bosques de coníferas del sur de Chile. Revista Chilena de Historia Natural 67: 209-220.

Gomes, L., Maracahipes, L., Marimon, B., Reis, S., Elias, F., Maracahipes-Santos, L., Marimon-Junior, B., Lenza, E. 2014. Post-fire recovery of savanna vegetation from rocky outcrops. Flora 209: 201-208.

Gomez-Gonzalez, S., Paula, S., Cavieres, L., Pausas, J.G. 2017. Postfire responses of the woody flora of central Chile: Insights from a germination experiment. Plos One 12(7).

González, M.E., Cortes, M., Gallo, L., Bekessy, S., Echeverría, C., Izquierdo, F., Montaldo, P. 2013. Coníferas chilenas: Araucaria araucana. En: Donoso, C. (ed.), Las especies arbóreas de los bosques templados de Chile y Argentina. Autoecología, pp. 36-53. Marisa Cuneo Ediciones, Valdivia, Chile.

González, M.E., Donoso, C., Ovalle, P., Martínez-Pastur, G. 2006. Nothofagus pumilio. En: Donoso, C. (ed.), Las especies arbóreas de los bosques templados de Chile y Argentina. Autoecología, pp. 486-500. Marisa Cuneo Ediciones, Valdivia, Chile.

González, M.E., Lara, A. 2015. Large fires in the Andean Araucaria forests: When a natural ecological process becomes a threat. Oryx 49: 389-395.

González, M.E., Lara, A., Urrutia, R., Bosnich, J. 2011. Climatic change and its potential impact on forest fire occurrence in south-central Chile (33 degrees-42 degrees S). Bosque 32: 215-219.

González, M.E., Szejner, M., Muñoz, A., Silva, J. 2010. Incendios catastróficos en bosques andinos de AraucariaNothofagus: Efecto de la severidad y respuesta de la vegetación. Revista Bosque Nativo 46: 12-17.

González, M.E., Veblen, T.T. 2006. Climatic influences on fire in Araucaria araucana-Nothofagus forests in the Andean cordillera of south-central Chile. Ecoscience 13: 342-350.

Gonzélez, M.E., Veblen, T.T., Sibold, J. 2005. Fire history of Araucaria-Nothofagus forests in Villarrica National Park, Chile. Journal of Biogeography 32: 1187-1202.

Hoffmann, W.A., Moreira, A.G. 2002. The role of fire in population dynamics of woody plants. In: OliveIrA, O.S., Marquis, R.J. (eds.), Cerrados of Brazil, pp. 159-177. Columbia University Press, New York, USA.

KeEley, J.E. 2006. Fire management on invasive plants in the Western United States. Conservation Biology 20: 375-384.

Key, C.H., Benson, N.C. 1999. Measuring and remote sensing of burn severity. In: NEUENSCHWANDER, L.F., RYAN, K.C. (eds.) Proceedings of the joint fire science conference. University of Idaho and International Association of Wildland Fire. $284 \mathrm{pp}$.

Kitzberger, T., Perry, G., Paritsis, J., Gowda, J., Tepley, A., Holz, A., Veblen, T. 2016. Fire-vegetation feedbacks and alternative states: Common mechanisms of temperate forest vulnerability to fire in southern South America and New Zealand. New Zealand Journal of Botany 54: 247-272.

Köppen, W. 1948. Climatología. Con un estudio de los climas de la tierra. Fondo de Cultura Económica, México D.F., México. 478 pp.

Lamont, B., Downes, K. 2011. Fire-stimulated flowering among resprouters and geophytes in Australia and South Africa. Plant Ecology 212: 2111-2125.

Le Maitre, D., Jones, C., Forsyth, G. 1992. Survival of eight woody sprouting species following an autumn fire in Swartboskloof, Cape Province, South Africa. South African Journal of Botany 58: 405-413.

Libano, A.M., Felfili, J.M. 2006. Mudanças temporais na composição florística e na diversidade de um cerrado sensu stricto do Brasil Central em um período de 18 anos (1985-2003). Acta Botanica Brasilica 20: 927-936.

Luebert, F., Pliscoff, P. 2006. Sinopsis bioclimática y vegetacional de Chile. Editorial Universitaria, Santiago, Chile. 316 pp.

Mack, M.C., D’Antonio, C.M. 1998. Impacts of biological invasions on disturbance regimes. Trends in Ecology \& Evolution 13: 195-198.

Matthei, O. 1995. Manual de las malezas que crecen en Chile. Alfabeta Impresores, Santiago, Chile. 545 pp.

Molina, J.R., Moreno, N., Moreno, R. 2017. Influence of fire regime on forest structure and restoration of a native forest type in the southern Andean range. Ecological Engineering 102: 390-396.

Montenegro, G., Ginocchio, R., Segura, A., Keely, J., Gómez, M. 2004. Fire regimes and vegetation responses in two Mediterranean climate regions. Revista Chilena de Historia Natural 77: 445-464.

Mora, M., Crisóstomo, R. 2016. Incendios forestales: Bajo el ojo de la teledetección. Revista Chile Forestal. Informe Técnico $N^{\circ} 383$, Corporación Nacional Forestal (CONAF), Santiago, Chile. 62 p.

Morgan, P., Moy, M., Droske, C.A., Lewis, S.A., Lentile, L.B., Robichaud, P.R., Hudak, A.T., Williams, C.J. 2015. Vegetation response to burn severity, native grass seeding, and salvage logging. Fire Ecology 11: 31-58.

Ojeda, F., Budde, K., Heuertz, M., Segarra-Moragues, J., GonzÁlez-Martínez, S. 2016. Biogeography and evolution of seeder and resprouter forms of Erica coccinea (Ericaceae) in the fire-prone Cape fynbos. Plant Ecology 212: 751-761.

Paritsis, J., Veblen, T., Holz, A. 2015. Positive fire feedbacks contribute to shifts from Nothofagus pumilio forests to fire-prone shrublands in Patagonia. Journal of Vegetation Science 26: 89-101.

Pauchard, A., Alaback, P. 2004. Influence of elevation, land use, and landscape context on patterns of alien plant invasions along roadsides in protected areas of south-central Chile. Conservation Biology 18: 238-248.

Peña, E., Valenzuela, L. 2008. Incremento de los incendios forestales en bosques naturales y plantaciones forestales en Chile. En: GonzÁlez-CABÁn, A. (ed.) Segundo simposio internacional sobre políticas, planificación y economía de los programas de protección contra incendios forestales: Una visión global. Departamento de Agricultura de EE.UU., Servicio Forestal, Estación de Investigación del Pacífico Suroeste. $612 \mathrm{p}$.

Pfab, M., Witkowski, E. 1999. Fire survival of the Critically Endangered succulent, Euphorbia clivicola R.A. Dyer fire-avoider or fire-tolerant? African Journal of Ecology 37: 249-257.

Pickett, S.T., Collins, S.L., Armesto, J.J. 1987. A hierarchical consideration of causes and mechanisms of succession. Vegetatio 69: 109-114.

R CoRe TeAm. 2016. R: A language and environment for statistical computing. $\mathrm{R}$ foundation for statistical computing, Vienna, 
Diferencias florísticas en distintas severidades de fuego: URRUTIA-EstradA, J. ET AL.

Austria. https://www.r-project.org

Raffaele, E., Veblen, T.T., Blackhall, M., Tercero-Bucardo, N. 2011. Synergistic influences of introduced herbivores and fire on vegetation change in northern Patagonia, Argentina. Journal of Vegetation Science 22: 59-71.

Riedemann, P., Aldunate, G. 2004. Flora nativa de valor ornamental. Identificación y propagación. Zona Sur. Editorial Andrés Bello, Santiago, Chile. 516 pp.

Rossi, J.P. 2011. Rich: An R package to analyse species richness. Diversity 3: 112-120.

Saha, S., Kuehne, C., Bauhus, J. 2017. Lessons learned from oak cluster planting trials in central Europe. Canadian Journal of Forest Research 47: 139-148.

Savadogo, P., Sawadogo, L., Tiveau, D. 2007. Effects of grazing intensity and prescribed fire on soil physical and hydrological properties and pasture yield in the savanna woodlands of Burkina Faso. Agriculture, Ecosystems \& Environment 118: 80-92.

Sinkins, P.A., OTfinowski, R. 2012. Invasion or retreat? The fate of exotic invaders on the northern prairies, 40 years after cattle grazing. Plant Ecology 213: 1251-1262.

Supp, S.R., ERNeSt, S.K.M. 2014. Species-level and communitylevel responses to disturbance: A cross-community analysis. Ecology 95: 1717-1723.

Teillier, S., Marticorena, A., Macaya, J., Bonnemaison, C., Delaunoy, J. 2014. Flora Reserva Biológica Huilo-Huilo. Guía para la identificación de las especies. Volumen I, II y
III. Fundación Huilo Huilo, Santiago, Chile. 473+343+373 pp.

Ubeda, X., Sarricolea, P. 2016. Wildfires in Chile: A review. Global and Planetary Change 146: 152-161.

Urrutia, J., Pauchard, A., García, R. 2013. Diferencias en la composición vegetal de un bosque de Araucaria araucana (Molina) K. Koch y Nothofagus antarctica (G. Forst.) Oerst. asociadas a un gradiente de invasión de Pinus contorta Douglas ex Loudon. Gayana Botánica 70: 127-135.

Zamorano-Elgueta, C., Cayuela, L., González-Espinosa, M., Lara, A., Parra-VÁzquez, M.R. 2012. Impacts of cattle on the South American temperate forests: Challenges for the conservation of the endangered monkey puzzle tree (Araucaria araucana) in Chile. Biological Conservation 152: 110-118.

Zhang, J., Mayor, S., He, F. 2014. Does disturbance regime change community assembly of angiosperm plant communities in the boreal forest? Journal of Plant Ecology 7: 188-201.

Zouhar, K., Smith, J.K., Sutherland, S., Brooks, M.L. 2008. Wildland fire in ecosystems. Fire and nonnative invasive plants. Department of Agriculture, Forest Service, Rocky Mountain Research Station, Ogden, Utah, USA. 355 pp.

Zuloaga, F.O., Morrone, O., Belgrano, M. 2008. Catálogo de las plantas vasculares del cono sur (Argentina, sur de Brasil, Chile, Paraguay y Uruguay). Vol. 1. Pteridophyta, Gymnospermae y Monocotyledoneae. Missouri Botanical Garden Press, Saint Louis, Missouri, USA. 3384 pp.

ANEXo 1. Listado de todas las plantas vasculares registradas en el área de estudio y en todos los niveles de severidad de fuego. FV: forma de vida. BNQ: bosque no quemado, BS: baja severidad, MS: media severidad, AS: alta severidad. El asterisco corresponde a especies exóticas. / List of all vascular plants registered in the study area across all fire-severity levels. FV: life form. BNQ: unburned forest, BS: low severity, MS: medium severity, AS: high severity. Asterisk corresponds to exotic species.

\begin{tabular}{|c|c|c|c|}
\hline Clase / Nombre Científico & FAMILIA & $\mathrm{FV}$ & $\begin{array}{c}\text { PRESENCIA EN NIVEL DE } \\
\text { SEVERIDAD }\end{array}$ \\
\hline \multicolumn{4}{|l|}{ PINOPSIDA } \\
\hline Araucaria araucana (Molina) K. Koch. & Araucariaceae & Árbol & BNQ, BS, MS, AS \\
\hline \multicolumn{4}{|l|}{ MAGNOLIOPSIDA } \\
\hline Acaena ovalifolia Ruiz \& Pav. & Rosaceae & Hierba & BNQ, BS, MS \\
\hline Acaena pinnatifida Ruiz \& Pav. & Rosaceae & Hierba & $\mathrm{BS}$ \\
\hline Adenocaulon chilense Less. & Asteraceae & Hierba & $\mathrm{BNQ}, \mathrm{BS}$ \\
\hline Adesmia emarginata $\mathrm{Clos}$ & Fabaceae & Arbusto & AS \\
\hline Anemone antucensis Poepp. & Ranunculaceae & Hierba & BS \\
\hline Azara alpina Poepp. \& Endl. & Salicaceae & Arbusto & BNQ, BS, MS, AS \\
\hline Baccharis magellanica (Lam.) Pers. & Asteraceae & Arbusto & AS \\
\hline Berberis microphylla G. Forst. & Berberidaceae & Arbusto & BNQ, BS, MS, AS \\
\hline Berberis serratodentata Lechl. & Berberidaceae & Arbusto & BNQ, BS, MS \\
\hline Berberis trigona Poepp. \& Endl. & Berberidaceae & Arbusto & $\mathrm{BNQ}, \mathrm{BS}, \mathrm{MS}$ \\
\hline Calceolaria valdiviana Phil. & Calceolariaceae & Hierba & BS \\
\hline Cerastium arvense L. * & Caryophyllaceae & Hierba & BNQ \\
\hline Cirsium arvense (L.) Scop. * & Asteraceae & Hierba & BS \\
\hline Galium hypocarpium (L.) Endl. \& Griseb. & Rubiaceae & Hierba & BNQ \\
\hline
\end{tabular}


Gayana Bot. 75(2), 2018

\begin{tabular}{|c|c|c|c|}
\hline Clase / Nombre Científico & FAMILIA & FV & $\begin{array}{c}\text { PRESENCIA ENNIVEL DE } \\
\text { SEVERIDAD }\end{array}$ \\
\hline \multicolumn{4}{|l|}{ MAGNOLIOPSIDA } \\
\hline Gaultheria poeppigii DC. & Ericaceae & Arbusto & BNQ, BS, MS, AS \\
\hline Hypochaeris radicata L. * & Asteraceae & Hierba & BS \\
\hline Lactuca serriola L. * & Asteraceae & Hierba & $\mathrm{BS}$ \\
\hline Lagenophora hariotii Franch. & Asteraceae & Hierba & BNQ \\
\hline Maytenus disticha (Hook. f.) Urb. & Celastraceae & Arbusto & BNQ, BS, MS \\
\hline Myoschilos oblongum Ruiz \& Pav. & Santalaceae & Arbusto & BNQ, BS \\
\hline Nothofagus pumilio (Poepp. \& Endl.) Krasser & Nothofagaceae & Árbol & BNQ, BS, MS \\
\hline Osmorhiza chilensis Hook. \& Arn. & Apiaceae & Hierba & BNQ, BS \\
\hline Perezia nutans Less. & Asteraceae & Hierba & BS \\
\hline Perezia pedicularidifolia Less. & Asteraceae & Hierba & BNQ \\
\hline Perezia prenanthoides Less. & Asteraceae & Hierba & $\mathrm{BNQ}, \mathrm{BS}$ \\
\hline Phacelia secunda J.F. Gmel. & Boraginaceae & Hierba & $\mathrm{BS}$ \\
\hline Quinchamalium chilense Molina & Schoepfiaceae & Hierba & BNQ \\
\hline Ribes cucullatum Hook. \& Arn. & Grossulariaceae & Arbusto & BS \\
\hline Ribes magellanicum Poir. & Grossulariaceae & Arbusto & BNQ, BS, MS \\
\hline Ribes trilobum Meyen & Grossulariaceae & Arbusto & $\mathrm{BNQ}, \mathrm{BS}$ \\
\hline Rubus geoides $\mathrm{Sm}$. & Rosaceae & Hierba & $\mathrm{BNQ}, \mathrm{BS}$ \\
\hline Rumex acetosella L. * & Polygonaceae & Hierba & $\mathrm{BS}, \mathrm{AS}$ \\
\hline Senecio pilquensis H. Buek & Asteraceae & Hierba & BNQ, MS \\
\hline Solanum valdiviense Dunal & Solanaceae & Arbusto & $\mathrm{MS}, \mathrm{AS}$ \\
\hline Veronica serpyllifolia L. * & Plantaginaceae & Hierba & $\mathrm{BS}$ \\
\hline Vicia nigricans Hook. \& Arn. & Fabaceae & Hierba & BNQ, BS, MS, AS \\
\hline Viola reichei Skottsb. & Violaceae & Hierba & BNQ, BS \\
\hline \multicolumn{4}{|l|}{ LILIOPSIDA } \\
\hline Agrostis capillaris L. * & Poaceae & Hierba & MS \\
\hline Alstroemeria aurea Graham & Alstroemeriaceae & Hierba & BNQ, BS, MS, AS \\
\hline Chusquea culeou E. Desv. & Poaceae & Arbusto & BNQ, BS, MS, AS \\
\hline Codonorchis lessonii (Brongn.) Lindl. & Orchidaceae & Hierba & BNQ \\
\hline Dioscorea reticulata Gay & Dioscoreaceae & Hierba & BNQ, AS \\
\hline Gavilea lutea (Pers.) M.N. Correa & Orchidaceae & Hierba & $\mathrm{BNQ}, \mathrm{BS}$ \\
\hline Holcus lanatus L. * & Poaceae & Hierba & BS \\
\hline Rhodophiala andicola (Poepp.) Traub. & Amaryllidaceae & Hierba & AS \\
\hline Sisyrinchium arenarium Poepp. & Iridaceae & Hierba & BNQ \\
\hline Uncinia scabriuscula G.A. Wheeler & Cyperaceae & Hierba & BNQ, BS, MS \\
\hline
\end{tabular}

Recibido: 10.10.2017

Aceptado: 07.08.2018 